Multiple radar targets detection by exploiting induced amplitude modulation.

IEEE Transactions on Signal Processing, 52, 4 (Apr. 2004), 903-913.

[46] Hi.zip, IPIX radar clutter data Available: http://soma.ece.mcmaster.ca/ipix/dartmouth/ datasets.html.

[47] Gerlach, K.

The effects of signal contamination on two adaptive detectors.

IEEE Transactions on Aerospace and Electronic Systems, 31, 1 (Jan. 1995), 297-309.

\section{Scheduling Beams with Different Priorities on a Military Surveillance Radar}

The problem of scheduling the searching, verification, and tracking tasks of a ground based, three-dimensional military surveillance radar is studied. Although the radar is mechanically steered in the sense that a servomechanism rotates the antenna at a constant turn rate, it has limited electronic steering capability in azimuth. The scheduling problem arises within a planning period during which the antenna scans a given physical range. A task/job corresponds to sending a transmission beam to hit a particular target. Targets are allowed to be hit with an angular deviation up to a predetermined magnitude. The steering mechanism of the radar helps alter these deviations by imposing a scan-off angle from broadside on the transmission beam. A list of jobs along with their priority weights, processing times, and ideal beam positions are given during a predetermined planning period. The ideal beam position for a given job allows hitting the corresponding target with zero deviation. Each job also has a set of available scan-off angles. It is possible to map the antenna's physical position, beam positions, scan-off angles, and angular deviations to a time scale. The goal is to select the subset of jobs to be processed during the given planning period and determining the starting time and scan-off angle for each selected job. The objectives are to simultaneously minimize the weighted number of unprocessed jobs and the total weighted deviation. An integer programming model and two versions of a heuristic mechanism that relies on the exact solution of a special case are proposed. Results of a computational study are presented.

\section{INTRODUCTION}

Radars are radio detection and ranging systems that transmit and receive electromagnetic waves - either microwaves or radio waves - to determine various attributes of distant objects such as location, speed, and direction of movement. Radars are used for a variety of purposes including weather forecasting, air traffic control, speed controls in ground traffic, and a range of military applications. Research and development in the supporting electronics technologies has led to many improvements in radar systems since their early introduction in the beginning of the twentieth century.

Manuscript received March 26, 2010; revised December 23, 2010 and April 6, 2011; released for publication May 17, 2011.

IEEE Log No. T-AES/48/2/943848.

Refereeing of this contribution was handled by T. Robertazzi. 


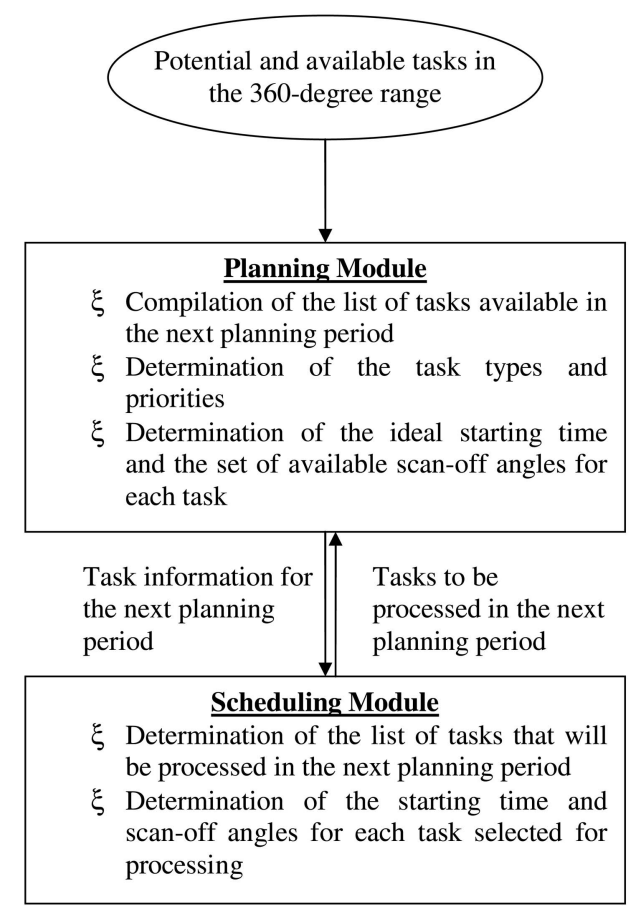

Fig. 1. Modules of resource management system.

The increasing complexity of radar systems utilized particularly in military operations resulted in a requirement for scheduling mechanisms specifically designed for radar operations. The expected positive impact of the effective solution of the arising scheduling problems on the actual physical performance of the radar, usually measured in terms of the root mean squared error (RMSE) of azimuth and elevation angles, stimulated much research in this area. This particular study is motivated by the need for developing a scheduling system to plan for the searching, verification, and tracking tasks of a ground based, mechanically steered, three dimensional (3D) military surveillance radar with limited electronic scanning capability in azimuth. The radar transmits beams to continuously perform trackwise scans over a 360-deg range within its search volume. The resource management system is composed of two different modules, the planning module and the scheduling module as shown in Fig. 1. Similar systems are seen in other applications for which Ding [4] provides a survey and a categorization. His general characterization of the multi-function radar resource management module closely resembles our application. Although the problem addressed in this paper falls into the scope of the scheduling module, we provide a brief description of the tasks carried out by the planning module to clarify the context in which the problem arises. For a more general overview of the resource management systems utilized in radar/sensor applications, the interested reader is referred to the recent works by Miranda, et al. [12] and Moran, et al. [13]. The planning module in our application takes the data collected on the position/timing of the available targets in the previous planning period and processes this data to produce a job list for the next planning period. The planning module is composed of several routines that are responsible for different aspects of the resource management system. The first step is to divide the planning period corresponding to a full $360-\mathrm{deg}$ rotation of the radar into successive scanning regions of a fixed size (e.g. 24 15-deg scanning regions). Then a tracking routine compiles a list of available tracking tasks in the next scanning region. Subsequently, search tasks are planned to enable scanning of the entire region covered by the radar. The verification tasks are added to the task list in an instantaneous manner as soon as a requirement arises. Once the full task list composed of search, tracking, and verification jobs is compiled, another routine determines the task priorities based on task types and whether or not a given task was unprocessed in a previous scan. Since the radar rotates at a constant speed, the unassigned radar jobs reappear in succeeding rotations of the radar. When an unassigned track and search job reappears in a given scanning region, their priorities are set higher according to a standard procedure. The unassigned verification tasks are neglected from further consideration in succeeding rotations due to the expectation that they either correspond to probable false alarms or else they reappear in the form of new high priority verification tasks for a real target. After the priorities are assigned, task durations are determined to guarantee a threshold detection probability for each task. A procedure utilized in this particular application exploits the manner in which the radar performs electronic scan-offs to produce a similar set of scan-off angles for each job independently of the task type. Although the confidentiality restrictions deter us from providing any details about the generation of the scan-off angles, a possible radar structure that allows their generation could be a system with a low number of bits in its phase shifters resulting in scan-off only in large increments of azimuth. Once all planning activities are completed, the problem for the next scanning region is solved by the scheduling routine producing the starting times and scan-off angles of the tasks to be completed within this scan region. The execution of the schedule provides the planning module with a feedback mechanism in the sense that the powers of the received beams reveal azimuth and elevation measurements that are used by the tracking routine. This procedure repeats itself in successive radar scans.

Note that different functions of the planning module consider different measures of performance. For instance, the false alarm rate is determined for this particular type of radar so as to ensure a reliable 
performance in terms of the detection of the real targets. Similarly, the task durations are determined based on a certain detection probability within a waveform determination routine. In a different context only the power measurements obtained from the received beams above a threshold detection probability are used within a direction finding routine to obtain the radar measurements guiding the track determination activity.

Returning to the functions of the scheduling module, each job in the list provided by the planning module has a given priority, a processing time, an ideal beam position, and an associated set of available scan-off angles (positive or negative) from broadside. A single job solely engages the radar for the duration of its processing time. It is permissible to process a job with a deviation from its ideal beam position as long as this deviation remains within certain bounds. Selection of a proper scan-off angle may help respecting these bounds by resulting in a reduction in the actual deviation. The scheduling model is responsible for the selection of the jobs to be performed in each planning period as well as the determination of their starting times and scan-off angles. The scan-off angles enable manipulation of the resulting deviations by imposing a time offset on the starting time of the transmission beams. Several aspects of the problem distinguish it from similar problems previously studied in the literature. First, the limited scanning capability of the radar in the azimuth alongside its mechanical rotation allows for the generation of scan-off angles, which are not available in other studies. Second, the problem allows the jobs to be completed with a deviation from their ideal starting times or not to be completed at all, and hence the tradeoffs in this regard need to be explicitly accounted for. Third, the proposed objective function to be optimized utilizes the task priorities to ensure that many critically important jobs are completed with small deviation. Although the problem is related to the two classical single machine scheduling problems with the objectives of minimizing the weighted number of tardy jobs (e.g., Potts and Van Wassenhove [16], Villarreal and Bulfin [17], and M'Hallah and Bulfin [9]) and minimizing the total earliness and tardiness penalties (Baker and Scudder [1]), the effect of the scan-off angles presents a novel feature, that to the best knowledge of the present authors, has not been investigated in the literature.

The remainder of the paper focuses on this new problem. The review of the most related research is presented in Section II. After presenting a formal definition of the problem, a mathematical model is given in Section III. Section IV proposes two heuristic mechanisms that start with a zero deviation solution and attempt to improve it by allowing deviation. Computational results on both random experiments and typical scenarios are given in Section V. Finally, Section VI concludes the paper.

\section{RELEVANT LITERATURE}

Although there have been a large number of studies on structural development of radars, there has not been as much research in the area of real time management of the radar resources to perform the associated tasks. This particular area started attracting some attention with the introduction and increasing popularity of phased-array radars. These more complex systems eliminate the need for using dedicated equipment for different types of tasks by allowing a single radar to perform a wide range of tasks such as searching, tracking, and guiding. As a result of this increased complexity, it became necessary to design algorithms to effectively handle task prioritization, selection, and scheduling.

One of the earliest papers in this area is by Orman, et al. [15] who consider on-line stochastic scheduling of coupled radar tasks involved in the surveying, tracking, and missile guiding operations of an electronically steered military radar. The radar jobs are viewed as coupled-tasks composed of pulse transmission and pulse reception. These two tasks are separated by a given time interval whose length is primarily determined by the distance of the target from the radar. Jobs have known processing times, priorities, and due start windows. The search volume is divided into search regions, and heuristic algorithms are proposed to schedule the available jobs within each region so as to maximize the radar utilization while considering job priorities.

Feinberg, et al. [6] consider a scheduling problem arising in the context of a nonrotating radar with electronic scanning capabilities. They propose an exact dynamic programming mechanism and several heuristics to investigate whether or not a feasible schedule that satisfies the dwell and revisit time constraints can be found. They perform a computational study to assess the performance of the proposed algorithms in terms of the percentage of randomly generated instances for which each algorithm finds a feasible solution. As an additional performance measure, they also consider the corresponding average cycle time (period duration) for the generated schedules.

Huizing and Bloemen [7] consider a multifunction radar with a single rotating or multiple fixed-phased array antennas that serve for both surveillance and missile support functions. They propose an efficient greedy constructive heuristic mechanism to schedule tasks in accordance with their priorities and transmission windows so as to use the energy and time resources in an effective manner. They report simulation results to assess the performance of their algorithm. 
De Jong and Van Norden [3] use metaheuristic techniques for the problem of scheduling search, track, midcourse, and terminal illumination tasks of a single multifunction radar. In particular they propose the use of evolutionary algorithms and their hybrid with fuzzy Lyapunov algorithm (Margialot and Langholz [8]). They test the performance of the proposed mechanisms on a simulated maritime scenario with insufficient capacity to execute all required tasks. They conclude that the hybrid algorithm tends to outperform the constituent algorithms.

Elshafei, et al. [5] consider the problem of maximizing the number of tracking tasks to be processed in the part of the planning horizon not used by the surveillance tasks. This objective requires interleaving as many other pulses as possible between the already scheduled transmission and reception pulses. The authors propose a Lagrangean relaxation procedure based on a new integer programming formulation.

Wintenby and Krishnamurthy [18] acknowledge the stochastic nature of radar tasks and view the resource management for adoptive airborne surveillance radars as a stochastic control problem. They formulate an instantaneous nominal utility objective that simultaneously accounts for target priority, accuracy, and cost of resource usage. They first model the problem as a Markov decision process whose computational requirements prove formidable. Then they propose an approximate solution procedure based on hierarchical time decomposition and Lagrangean relaxation.

Miranda, et al. [10] review scheduling and task prioritization as two related aspects of radar resource management. Miranda, et al. [11] further focus on the scheduling aspect, and compare the performance of two scheduling algorithms previously proposed in the literature for multifunction radar systems. These two algorithms developed by Orman, et al. [15] and Butler [2] are tested with a simulation model under various settings. The main conclusion is that the two algorithms perform similarly under a heavy load where the radar lacks sufficient resources to fulfill all available tasks. However, when the radar operates with an underload, the Butler algorithm results in better resource utilization.

In a recent study, Winter and Baptiste [19] consider a problem of scheduling the research, tracking, data link, and calibration tasks of an electronically steered airborne radar. Since an electronically steered radar has the ability to emit agile beams whose direction and waveform can be instantaneously adjusted, it can handle different task types consecutively. The authors list several technical constraints such as task nonoverlapping, not losing a tracked target, playing data link dwells at regular intervals, and a limit on the minimum number of research dwells. The objective is to minimize the total penalty assessed based on the distance between starting times of the operations belonging to a given job defined as a single radar function that must be repeated in a somewhat strict manner. After showing that the general problem is NP-hard, the authors develop a linear programming model to determine the starting times for the special case in which the sequence of operations is fixed. In addition they propose a local search algorithm and two lower bounds for the original problem.

Once again the problem studied in our paper is different from these studies in several ways. First, the electronic scan-off capability of the radar in the azimuth alongside its mechanical rotation makes it possible to use scan-off angles, and selection of an appropriate scan-off angle is also a part of our problem. Second, our problem allows for processing jobs with deviation, thus we consider the tradeoff between deviation and number of jobs processed. Finally, we use job priorities within the scheduling problem, and attempt to support doing important jobs with smaller deviation. In the next section we formally define and formulate this new problem.

\section{PROBLEM DEFINITION AND MATHEMATICAL FORMULATION}

This section formally defines the research problem and develops a mixed integer programming model for its solution. Integer programming formulations and their optimization methodologies are covered in depth in the fundamental resources by Nemhauser and Wolsey [14] and Wolsey [20]. Section IIIA aims to describe the mechanism with which the physical activities of the radar within a scanning range can be mapped into a scheduling problem over the time scale in the corresponding planning period. Section IIIB presents the necessary mathematical notation and develops an integer programming formulation for the scheduling problem.

\section{A. Problem Definition}

The radar continuously rotates at a constant speed and processes the selected tasks within its physical range at any given position during its rotation. Since there may be a large number of tasks available for processing in the search volume covered by a full rotation, scheduling problems are defined for equally sized consecutive scan ranges. Fig. 2(a) illustrates the way in which the full 360-deg rotation of the radar is divided into a series of successive scan ranges. The light and dark colored arcs along the innermost circle represent the successive scan ranges. Fig. 2(b) provides a larger and more detailed view of a section of the ring shown in Fig. 2(a). It is possible to observe in this larger view that the other circles 


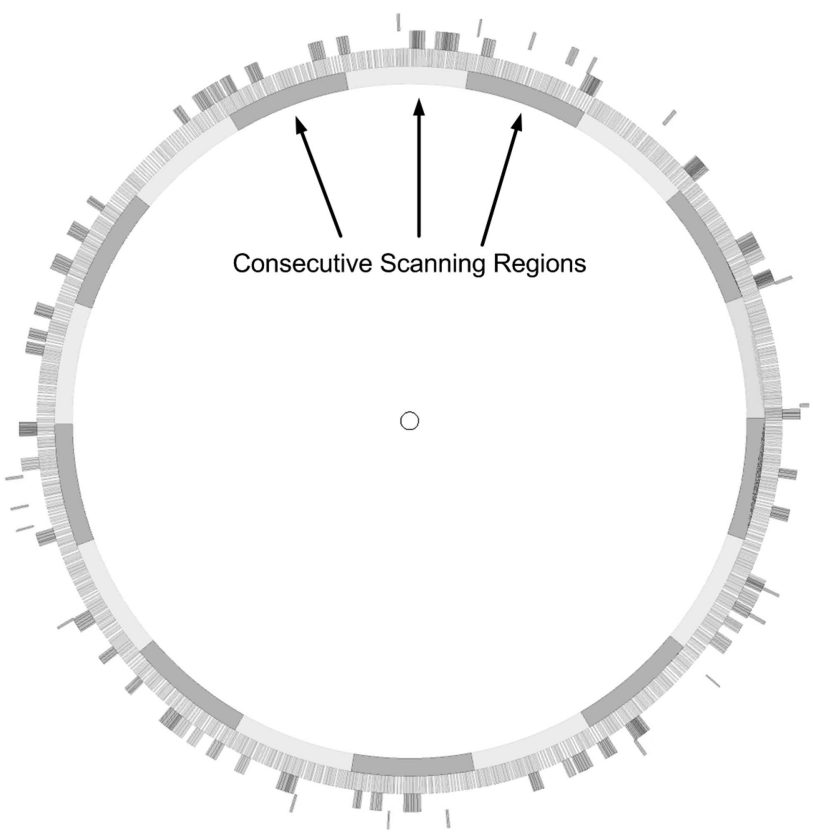

(a)

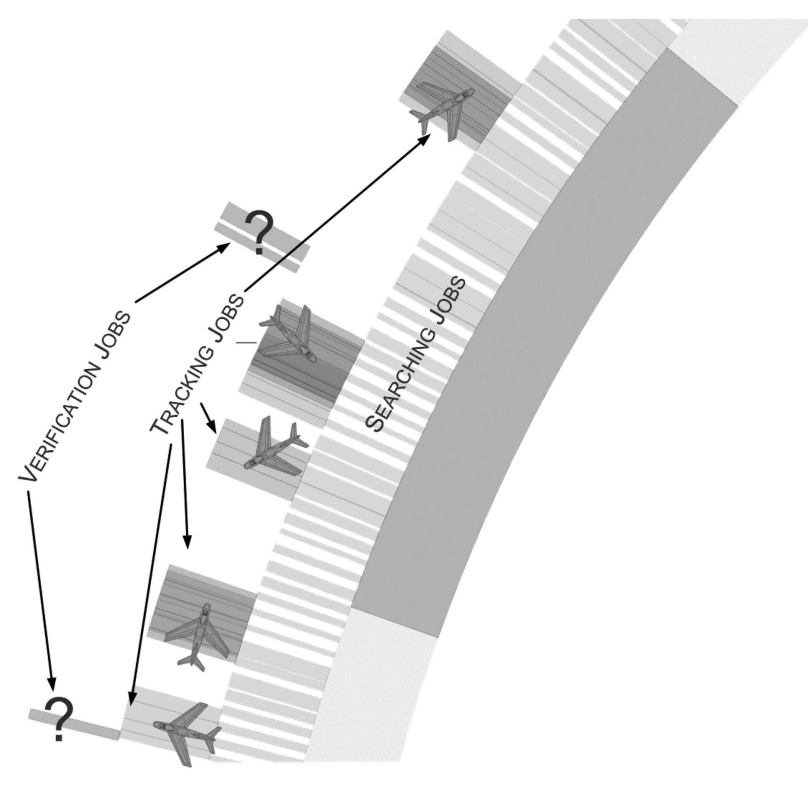

(b)

Fig. 2. Pictorial depiction of scanning ranges and radar jobs. (a) Scanning regions. (b) Jobs of different types.

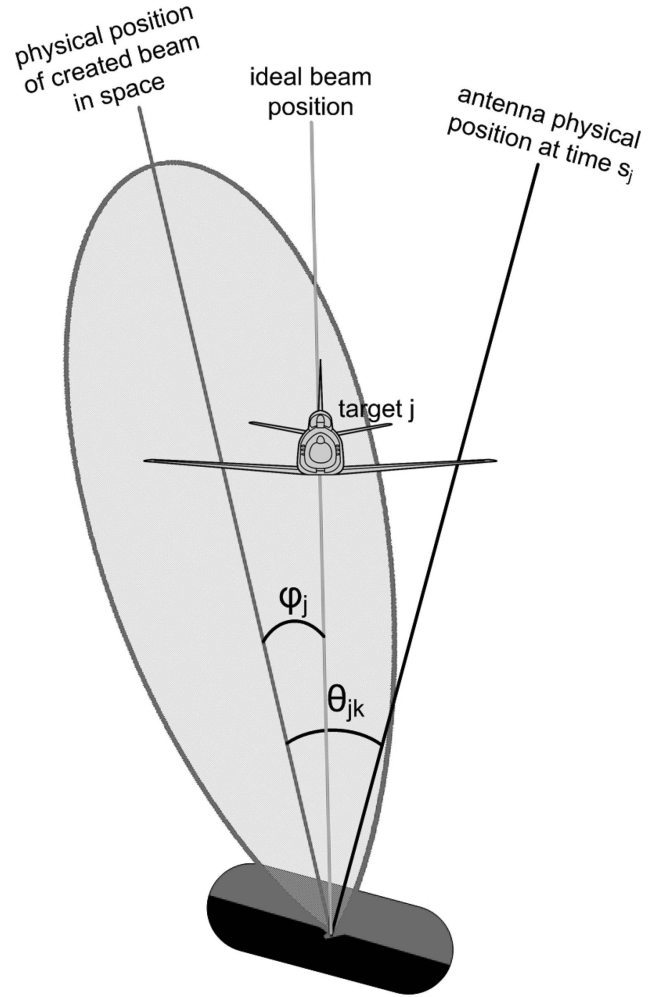

Fig. 3. Effect of scan-off angle and resulting angular deviation.

provide a representation of the search, tracking, and verification tasks in the order from inside out. Each scheduling problem considers the tasks (i.e., search, tracking, and verification jobs) within a single scan range. Fig. 3 shows the effect of scan-off angle on a transmission beam. In particular a target $j$ can be processed by sending a transmission beam at a certain starting time $s_{j}$ with a positive or negative scan-off angle $\theta_{j k}$. The scan-off angle imposes an angular offset that shifts the physical position of the created beam from the antenna's physical position (i.e., from broadside). If the created beam coincides with the ideal beam position, the target can be processed with zero deviation. Otherwise, an angular deviation $\varphi_{j}$ between the physical position of the created beam and the target's ideal beam position will be realized.

The ideal starting time $I_{j}$ is defined as the time at which the physical position of the antenna coincides with the ideal beam position. Suppose angles $\theta_{j k}$ and $\varphi_{j}$ are measured in degrees, and let $r$ denote the constant turn rate of the antenna in degrees per unit time. Then the time offset imposed by scan-off angle $\theta_{j k}$ is calculated as $a_{j k}=\theta_{j k} / r$. Similarly, the resulting angular deviation $\varphi_{j}$ corresponds to a deviation from the ideal starting time of $d_{j}=\left|\varphi_{j} / r\right|=\left|I_{j}-\left(s_{j}+a_{j k}\right)\right|$.

\section{B. Notation and Formulation}

Suppose there are $n$ jobs where a job indexed by $j$ has $m_{j}$ available scan-off angles. Each job selected for processing needs to be assigned a single scan-off angle that affects its deviation from the ideal starting time. All selected jobs need to be processed within the planning horizon in a nonpreemptive manner. At most one job can be processed in any given time interval. The objective is to simultaneously minimize both the weighted number of unprocessed jobs and the total weighted deviation of the processed jobs from their respective ideal starting times. 


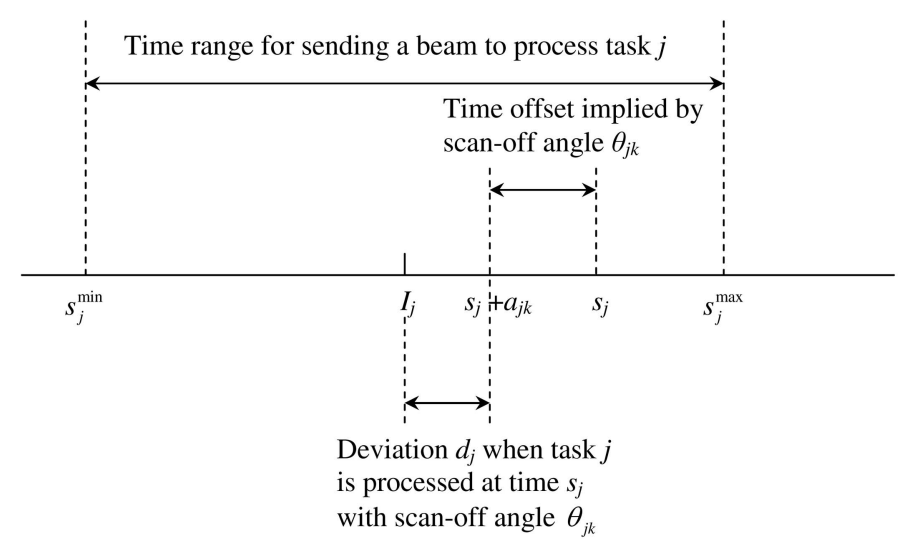

Fig. 4. Effect of scan-off angle and starting time on time deviation.

Define the following notation to facilitate a mathematical exposition.

$p_{j} \equiv$ Processing time of job $j$.

$I_{j} \equiv$ Ideal starting time of job $j$.

$w_{j} \equiv$ Priority weight of job $j$.

$m_{j} \equiv$ Number of available scan-off angles for job $j$.

$a_{j k} \equiv$ Time offset corresponding to scan-off angle $k$ for job $j$.

$D_{\max } \equiv$ Maximum permissible deviation for any job measured in time units.

$H \equiv$ Length of the planning horizon within which the last job must complete.

Assume without loss of generality that the scan-off angles for job $j$ are indexed such that $a_{j 1} \geq a_{j 2} \cdots \geq$ $a_{j m_{j}}$. The following theorem proves helpful in the mathematical formulation.

THEOREM 1 The starting time of job $j$ may not exceed

$$
s_{j}^{\max }=\max \left(0, \min \left(I_{j}-a_{j m_{j}}+D_{\max }, H-p_{j}\right)\right)
$$

and may not be any smaller than

$$
s_{j}^{\min }=\max \left(0, I_{j}-a_{j 1}-D_{\max }\right) .
$$

PROOF All jobs must be processed within the planning horizon. Consequently, $s_{j} \geq 0$ and $s_{j} \leq H-$ $p_{j}$. Furthermore, the maximum permissible deviation for any job $j$ is $D_{\max }$. That is,

$$
\begin{gathered}
\left|I_{j}-\left(s_{j}+a_{j k}\right)\right| \leq D_{\max } \\
-D_{\max } \leq I_{j}-\left(s_{j}+a_{j k}\right) \leq D_{\max } \\
I_{j}-a_{j k}-D_{\max } \leq s_{j} \leq I_{j}-a_{j k}+D_{\max } .
\end{gathered}
$$

Since the scan-off angles are indexed in descending order, we have that

$$
I_{j}-a_{j 1}-D_{\max } \leq s_{j} \leq I_{j}-a_{j m_{j}}+D_{\max } .
$$

The following three corollaries follow from Theorem 1.

COROLlaRY 1 When $s_{j}^{\min }>s_{j}^{\max }$, it is not feasible to process job $j$.

COROLlaRY 2 If both jobs $i$ and $j$ are to be processed and if $s_{i}^{\max } \leq s_{j}^{\min }$ then job $i$ must precede job $j$.

COROLlaRY 3 If $s_{i}^{\min }+p_{i}>s_{j}^{\max }$ then job i may not start processing before job $j$.

Note that those jobs whose processing is infeasible due to Corollary 1 can be eliminated through a preprocessing mechanism. Similarly, the feasibility conditions implied by Corollaries 2 and 3 can be taken into account during a preprocessing phase.

When the scan-off angle for a job to be processed is selected, the minimum and maximum starting times of that job can be calculated based on the maximum permissible deviation, $D_{\max }$. The following proposition calculates these quantities that will be used as a constraint on the starting time in the mathematical formulation.

PROPOSITION 1 The minimum and maximum starting times of job $j$ when processed with scan-off angle $k$ are $s_{j k}^{\min }=\max \left(0, I_{j}-a_{j k}-D_{\max }\right)$ and $s_{j k}^{\max }=\min (H-$ $\left.p_{j}, I_{j}-a_{j k}+D_{\max }\right)$, respectively.

Define the following decision variables for the mathematical model.

$s_{j}=$ Starting time of job $j$.

$d_{j}=$ Deviation of job $j$ from its ideal starting time.

$y_{i j}= \begin{cases}1 & \text { if job } i \text { is processed before job } j \\ 0 & \text { otherwise. }\end{cases}$

$x_{j k}=\left\{\begin{array}{ll}1 & \text { if job } j \text { is processed with scan-off angle } k \\ 0 & \text { otherwise. }\end{array}\right.$.

Fig. 4 illustrates the effect of the selected starting time $\left(s_{j}\right)$ and scan-off angle $\left(\theta_{j k}\right)$ combination on the deviation $\left(d_{j}\right)$ of job $j$. Note that if no scan-off angle is assigned to a given job, then that job is not selected 
for processing. That is, if $\sum_{k=1}^{m_{j}} x_{j k}=0$ then job $j$ is not processed.

The proposed model is as follows.

$$
\begin{aligned}
& \min \sum_{j=1}^{n} w_{j}\left(\alpha\left(1-\sum_{k=1}^{m_{j}} x_{j k}\right)+\beta d_{j}\right) \\
& \text { s.t. } \quad y_{i j}+y_{j i} \geq \sum_{k=1}^{m_{j}} x_{j k}+\sum_{k=1}^{m_{i}} x_{i k}-1 \quad \forall i \neq j \\
& y_{i j}+y_{j i} \leq \sum_{k=1}^{m_{j}} x_{j k} \quad \forall i \neq j \\
& s_{i}+p_{i} \leq s_{j}+\left(s_{i}^{\max }+p_{i}\right)\left(1-y_{i j}\right) \quad \forall i \neq j \\
& s_{j} \leq \sum_{k=1}^{m_{j}} s_{j k}^{\max } x_{j k} \quad \forall j \\
& s_{j} \geq \sum_{k=1}^{m_{j}} s_{j k}^{\min } x_{j k} \quad \forall j \\
& d_{j} \geq I_{j}-\left(s_{j}+\sum_{k=1}^{m_{j}} a_{j k} x_{j k}\right)-I_{j}\left(1-\sum_{k=1}^{m_{j}} x_{j k}\right) \quad \forall j \\
& d_{j} \geq\left(s_{j}+\sum_{k=1}^{m_{j}} a_{j k} x_{j k}\right)-I_{j}+I_{j}\left(1-\sum_{k=1}^{m_{j}} x_{j k}\right) \quad \forall j \\
& d_{j} \leq D_{\max } \forall j \\
& x_{j k}, y_{i j} \in\{0,1\} \quad \forall i, j, k \\
& s_{j}, d_{j} \geq 0 \quad \forall j .
\end{aligned}
$$

The objective function presented in (1) minimizes the additive value of the total weighted number of unprocessed jobs and the total weighted deviation of those selected for processing. Coefficients $\alpha$ and $\beta$, where $\alpha+\beta=1.0$, are nonnegative scalars given as problem parameters to reflect the relative importance of the two evaluation measures. Constraint sets (2) and (3) ensure for any two jobs both selected for processing that one must precede the other. Constraint set (4) allows a job to start its processing only after all of its preceding jobs are completed. Constraint sets (2), (3) and (4) together also imply that at most one scan-off angle can be assigned to any given job. Constraint sets (5) and (6) restrict the starting time of all jobs within the feasible limits determined according to Proposition 1. Constraint sets (7) and (8) calculate the deviation of job starting times from the corresponding ideal starting times. These constraints take into account the assigned scan-off angles in the calculation of these deviations. Finally, constraint sets (10) and (11) impose, respectively, binary and nonnegativity restrictions for the relevant decision variables.

\section{TWO-STAGE SOLUTION METHODOLOGY}

Although the formulation presented in the previous section models the scheduling problem at hand in its most accurate form, preliminary experimentation with realistically sized problems indicate that the best available solvers such as CPLEX fail to provide optimum solutions in reasonable computational times (e.g. $60 \mathrm{~min}$ ). Note that such a shortcoming is common in many challenging integer programming formulations arising in different contexts. Hence, it is justified to look for efficient heuristic mechanisms to find satisfactory solutions to the problem. In this section we develop a two-stage heuristic methodology that serves to this end. In particular we first consider a special case of the problem where jobs are only permitted to start exactly at their ideal starting times and then we take this first stage solution and postprocess it in the second stage in an attempt to improve the solution quality. In particular the goal is to exploit the potentially more modest computational requirements in the special case with no deviation to obtain a starting solution to be improved in the second stage.

The following two subsections detail the tasks handled in the two stages.

\section{A. First Stage: Special Case with No Deviation Allowed}

In this section we consider a special case of the problem in which the jobs are required to start at their respective ideal starting times. We develop a new and more compact model for this special case based on the following proposition.

PROPOSITION 2 Jobs $j$ and $i$ cannot be processed with the respective scan-off angles of $k$ and $l$ if the following two conditions hold simultaneously

$$
\begin{aligned}
& I_{j}-a_{j k} \geq I_{i}-a_{i l} \\
& I_{j}-a_{j k} \leq I_{i}-a_{i l}+p_{i} .
\end{aligned}
$$

PROOF If these conditions simultaneously hold, job $j$ starts its processing while the processing of job $i$ is still in progress.

Define $A_{j k}$ as the set of job and scan-off angle pairs which are infeasible due to Proposition 2 when job $j$ is to be processed with scan-off angle $k$. We propose the following mixed integer programming (MIP) model for this special case.

$$
\begin{array}{ll} 
& \min \\
& \sum_{j=1}^{n} w_{j}\left(1-\sum_{k=1}^{m_{j}} x_{j k}\right) \\
\text { s.t. } & \sum_{k=1}^{m_{j}} x_{j k} \leq 1 \quad \forall j \\
& x_{j k}+x_{i l} \leq 1 \quad \text { for } \quad i, j, k, l:(i, l) \in A_{j k} \\
& x_{j k} \in\{0,1\} \quad \forall j, k .
\end{array}
$$


The objective function (12) minimizes the total weighted number of unprocessed jobs. Constraint set (13) allows each job to be processed by at most one of the available scan-off angles. Constraint set (14) relies on the definition of set $A_{j k}$ to ensure a feasible assignment of scan-off angles to all job pairs.

The compact set packing nature of this model makes it possible to solve this special case in an efficient manner. The solution can be considered as a starting point that can be improved by allowing deviations in an improvement phase to potentially improve the bi-objective optimum value in the general problem. The following section proposes two alternative postprocessing mechanisms for this improvement stage.

\section{B. Second Stage: Allowing Deviation}

The approximate methods first solve the model of Section IVA to determine the optimum job and scan-off angle pairs that can be processed with no deviation. Then they incorporate the notion of processing certain jobs with a permissible amount of deviation to potentially introduce additional jobs into the schedule. Clearly, the goal is to improve the bi-objective value. Neither of the models consider excluding a job from the initial list of processed jobs, and they differ in terms of whether or not the scan-off angles for these fixed jobs can be changed in the improvement phase. We define $x_{j k}^{*}$ as the optimum value of each $x_{j k}$ in the first stage.

1) Fixed Jobs and Fixed Scan-off Angles: This section introduces the following second stage model that fixes the scan-off angle selection of the jobs scheduled with no deviation in the first stage problem.

$$
\begin{aligned}
& \max \sum_{j=1}^{n} w_{j}\left(\alpha\left(1-\sum_{k=1}^{m_{j}} x_{j k}\right)+\beta d_{j}\right) \\
& \text { s.t. } \quad x_{j k}=x_{j k}^{*} \quad \forall j, k: x_{j k}^{*}=1 .
\end{aligned}
$$

$$
\text { (2) }-(11)
$$

Constraint set (17) fixes the jobs selected to be processed in the first stage along with their scan-off angles.

2) Fixed Jobs and Variable Scan-off Angles: The approximation scheme of this section allows assignment of different scan-off angles to the jobs selected for processing in the optimum solution to the first stage problem. The only restriction imposed in the second stage is that a job selected for processing in the first stage cannot be excluded from the list of jobs to be processed. The resulting model is the same as in Section IVB1 with the replacement of constraints (17) by (19).

$$
\begin{aligned}
& \max \sum_{j=1}^{n} w_{j}\left(\alpha\left(1-\sum_{k=1}^{m_{j}} x_{j k}\right)+\beta d_{j}\right) \\
& \text { s.t. } \quad \sum_{k=1}^{m_{j}} x_{j k}=\sum_{k=1}^{m_{j}} x_{j k}^{*} \quad \forall j: \sum_{k=1}^{m_{j}}: x_{j k}^{*}=1 .
\end{aligned}
$$

$$
\text { (2)-(11) }
$$

Since this version allows greater flexibility in shifting the starting times of jobs, the optimum objective values cannot be any worse than those obtained from the method of Section IVB1. The potential improvements however may certainly come at the cost of reduced efficiency in solution.

\section{EXPERIMENTAL ASSESSMENT}

The performance of the proposed models is assessed through a computational study that involves both randomly generated problems and typical scenarios. In the following we first describe the mechanism of generating the random test problems, and then proceed with a presentation and evaluation of the computational results.

\section{A. Experimental Design}

Although the motivating application offers a collection of real problem instances, we primarily base our performance assessment on random experiments due to confidentiality restrictions. The random experiments vary the levels of certain key problem parameters in a systematic manner to produce a multitude of realistic scenarios to test the performance of the proposed methods with respect to both their effectiveness and computational efficiency.

The two objectives are assigned equal weights by setting $\alpha=\beta=0.5$. Problems with 25, 50, and 75 jobs are considered, each with $2,4,8$, and 16 scan-off angles. In line with the motivating application, the length of the planning period $H$ is set at $15 \mathrm{deg}$ and the maximum permissible deviation $D_{\max }$ is set at 0.5 . Ideal starting times are sampled uniformly between 0 and $H$. Processing times and scan-off angles are also expressed in degrees. The average processing time is set at $H / n$. To consider both low and high variability in job durations, the processing times are sampled from continuous uniform distributions with ranges of $[0.80 \times H / n, 1.20 \times H / n]$ and $[0.60 \times H / n, 1.40 \times$ $H / n]$. Since in practice scan-off angles tend to be similar for all jobs, this factor is not systematically varied. Instead, the scan-off angles are sampled from a continuous uniform distribution with a range between -2.0 and 2.0. Use of a continuous distribution is instrumental in realistically modeling the effect that changing the elevation creates on the scan-off angles.

The priority weights of the jobs are sampled in accordance with their respective job types. The 
TABLE I

Control Factors and their Levels

\begin{tabular}{cc}
\hline \hline Control Factor & Levels \\
\hline Number of jobs & $25,50,75$ \\
Number of scan-off angles & $2,4,8,16$ \\
Range of processing times & low, high \\
Scenario type & A, B \\
\hline
\end{tabular}

verification jobs are of the highest priority and they are assigned a weight of 10000 . The priority weights of tracking jobs are sampled uniformly over a range between 400 and 700 . The search jobs are of the lowest priority with uniform weights between 100 and 200 . The percentage of verification jobs is relatively stable at all times and it is set at $8 \%$. Two different types of scenarios (types A and B) are generated based on the relative frequency of the tracking jobs to the search jobs. Type A scenarios have 33\% tracking jobs leaving $59 \%$ for search jobs. These percentages are set at $2 \%$ and $90 \%$, respectively, for tracking and search jobs in type B scenarios.

Table I presents a summary of the control factors and their respective levels. All factor level combinations are considered in a full factorial design resulting in a total number of 48 treatments. Due to the stochastic elements in design, ten random instances are considered in each treatment. Therefore, this design includes a total of 480 problem instances. The following subsection reports on the performance of the proposed algorithms on both these instances and some typical scenarios.

\section{B. Computational Results}

All problems are solved on a $2.66 \mathrm{GHz} \mathrm{PC}$ with 8 GB of RAM using CPLEX 10.1. Due to the computational difficulty involved in the solution of the problem, each solution procedure is allowed to run for a maximum of $1 \mathrm{hr}$ for every instance. When the solution of any given instance cannot be completed in the alloted time, results are reported for the best integer solution obtained. Although the lower bounds obtained from the linear programming relaxations (allowing integer variables to take on fractional values) are known to be weak, the gaps from the best relaxed solutions that could be found by each strategy are also reported to provide an idea about the relative solution quality. All statements made about the results of the random experiments in this section are statistically supported at a significance level of 0.05 .

Table II shows the results with the formulation proposed in Section IVA for the special case with no deviation. The first two columns correspond to the problem characteristics as explained in Section VA. The objective value column shows the value of expression (12). Similarly, the next column presents the number of jobs which can be scheduled to start exactly on their ideal starting times. The number of nodes is a statistic reported by the CPLEX solver to indicate the size of the search tree at the termination of the algorithm. The CPU times listed in the next column provide further information on the computational effort. The final column lists the percentage gaps from the best available lower bound as reported by CPLEX. A percentage gap of zero indicates that the problem is solved to optimality. In rows the table reports the average figures for each level of all control factors. As seen in the table the proposed formulation is very efficient in solving the special case. In fact, 467 of the 480 attempted instances were solved well under the alloted time limit of $1 \mathrm{hr}$. The average CPU times for small and medium sized problems with up to 50 jobs and 8 scan-off angles are less than a half minute. The percentage of jobs processed remains stable with an average between $76 \%$ and $78 \%$ as the number of jobs is increased. Increasing the number of scan-off angles results in a decrease in the total penalty cost along with a respective increase in the average number of jobs processed. Increasing the range of processing times increases the average CPU time requirements. Other than the increased average penalty cost resulting from a larger percentage of more critical tracking jobs, no significant differences are observed between the results of the type A and type B scenarios. The standard deviations of the objective function value and number of jobs done are shown parenthetically. As expected, these figures are large due to the fact that we base our asessments on a wide variety of instances.

The no-deviation solutions are used as an initial integer feasible solution by the original formulation presented in Section IIIB. This formulation allows changing this initial solution in terms of both the selection of the jobs to be done and their scan-off angle assignments. That is, the provision of the initial solution is merely an attempt to speed up the convergence to an initial feasible solution. Table III shows the corresponding results. The format of the table is similar to that of Table II with the exception of the objective value column which now reports the value of expression (1). Consequently, the jobs reported in the number of jobs done column may include deviations from their ideal starting times.

Only 35 of the 480 instances in the problem set were solved to optimality within the alloted time with this formulation. For the remaining problems the best found integer feasible solution within the alloted time is reported, and hence the non-zero gaps. Smaller instances in terms of number of jobs and scan-off angles tend to require less computational time. Since the results for larger instances have large optimality gaps, we refrain from commenting on the factor effects on the objective function and on the number of jobs done. However, we acknowledge that these 
TABLE II

Summary of Results when no Deviation is Allowed

\begin{tabular}{|c|c|c|c|c|c|c|}
\hline Control Factor & Level & $\begin{array}{c}\text { Objective } \\
\text { Value }\end{array}$ & $\begin{array}{l}\text { Number of } \\
\text { Jobs Done }\end{array}$ & $\begin{array}{l}\text { Number of } \\
\text { Nodes }\end{array}$ & $\begin{array}{l}\text { CPU Time } \\
\quad(\mathrm{sec})\end{array}$ & $\begin{array}{l}\text { Gap } \\
(\%)\end{array}$ \\
\hline \multirow{3}{*}{$\begin{array}{l}\text { Number of } \\
\text { Jobs }\end{array}$} & 25 & $\begin{array}{c}603.24 \\
(405.41)\end{array}$ & $\begin{array}{l}19.00 \\
(2.79)\end{array}$ & 29.01 & 0.25 & 0.00 \\
\hline & 50 & $\begin{array}{c}1127.97 \\
(1253.05)\end{array}$ & $\begin{array}{l}39.04 \\
(5.73)\end{array}$ & 4000.83 & 19.87 & 0.00 \\
\hline & 75 & $\begin{array}{c}1746.64 \\
(1759.02)\end{array}$ & $\begin{array}{l}58.48 \\
(8.75)\end{array}$ & 42004.11 & 476.59 & 0.59 \\
\hline \multirow{4}{*}{$\begin{array}{c}\text { Number of } \\
\text { scan-off angles }\end{array}$} & 2 & $\begin{array}{c}2391.39 \\
(1758.99)\end{array}$ & $\begin{array}{c}30.92 \\
(12.83)\end{array}$ & 0.00 & 0.08 & 0.00 \\
\hline & & $\begin{array}{c}1376.77 \\
(1227.86)\end{array}$ & $\begin{array}{c}36.78 \\
(15.34)\end{array}$ & 2.39 & 0.13 & 0.00 \\
\hline & 8 & $\begin{array}{c}570.94 \\
(202.91)\end{array}$ & $\begin{array}{c}42.22 \\
(17.81)\end{array}$ & 617.52 & 2.22 & 0.00 \\
\hline & 16 & $\begin{array}{c}298.03 \\
(118.95)\end{array}$ & $\begin{array}{c}45.44 \\
(19.09)\end{array}$ & 60758.70 & 659.86 & 0.79 \\
\hline \multirow{2}{*}{$\begin{array}{c}\text { Range of } \\
\text { processing times }\end{array}$} & & $\begin{array}{c}1172.02 \\
(1353.51)\end{array}$ & $\begin{array}{c}38.59 \\
(17.21)\end{array}$ & 11293.69 & 116.01 & 0.04 \\
\hline & High & $\begin{array}{c}1146.55 \\
(1348.57)\end{array}$ & $\begin{array}{c}39.09 \\
(17.42)\end{array}$ & 19395.61 & 215.13 & 0.35 \\
\hline \multirow{2}{*}{$\begin{array}{c}\text { Scenario } \\
\text { type }\end{array}$} & A & $\begin{array}{c}1355.43 \\
(1503.01)\end{array}$ & $\begin{array}{c}38.56 \\
(17.24)\end{array}$ & 16638.76 & 170.26 & 0.22 \\
\hline & B & $\begin{array}{c}963.13 \\
(1146.58)\end{array}$ & $\begin{array}{c}39.12 \\
(17.39)\end{array}$ & 14050.54 & 160.88 & 0.17 \\
\hline
\end{tabular}

TABLE III

Summary of Results with the Original Formulation

\begin{tabular}{|c|c|c|c|c|c|c|}
\hline Control Factor & Level & $\begin{array}{l}\text { Objective } \\
\text { Value }\end{array}$ & $\begin{array}{l}\text { Number of } \\
\text { Jobs Done }\end{array}$ & $\begin{array}{c}\text { Number of } \\
\text { Nodes }\end{array}$ & $\begin{array}{l}\text { CPU Time } \\
\text { (sec) }\end{array}$ & $\begin{array}{l}\text { Gap } \\
(\%)\end{array}$ \\
\hline \multirow{3}{*}{$\begin{array}{l}\text { Number of } \\
\text { jobs }\end{array}$} & 25.00 & $\begin{array}{c}363.91 \\
(231.64)\end{array}$ & $\begin{array}{l}22.16 \\
(1.39)\end{array}$ & 1834240.32 & 2945.91 & 70.76 \\
\hline & 50.00 & $\begin{array}{c}808.51 \\
(1042.07)\end{array}$ & $\begin{array}{l}43.82 \\
(3.11)\end{array}$ & 595317.80 & 3600.66 & 93.60 \\
\hline & 75.00 & $\begin{array}{c}1390.89 \\
(1358.43)\end{array}$ & $\begin{array}{l}63.27 \\
(6.59)\end{array}$ & 257372.34 & 3600.17 & 93.10 \\
\hline \multirow{4}{*}{$\begin{array}{c}\text { Number of } \\
\text { latency angles }\end{array}$} & 2.00 & $\begin{array}{c}1489.24 \\
(1429.44)\end{array}$ & $\begin{array}{c}42.63 \\
(17.74)\end{array}$ & 565184.90 & 2728.55 & 54.38 \\
\hline & 4.00 & $\begin{array}{c}1134.55 \\
(1287.01)\end{array}$ & $\begin{array}{c}40.79 \\
(15.32)\end{array}$ & 1109160.18 & 3599.89 & 89.35 \\
\hline & 8.00 & $\begin{array}{c}507.67 \\
(231.63)\end{array}$ & $\begin{array}{c}43.25 \\
(17.06)\end{array}$ & 989521.52 & 3600.41 & 99.59 \\
\hline & 16.00 & $\begin{array}{c}286.29 \\
(126.23)\end{array}$ & $\begin{array}{c}45.66 \\
(18.92)\end{array}$ & 918707.34 & 3600.13 & 99.95 \\
\hline \multirow{2}{*}{$\begin{array}{c}\text { Range of } \\
\text { processing times }\end{array}$} & & $\begin{array}{c}869.49 \\
(1078.20)\end{array}$ & $\begin{array}{c}42.89 \\
(17.25)\end{array}$ & 905041.26 & 3389.23 & 85.92 \\
\hline & High & $\begin{array}{c}839.39 \\
(1085.34)\end{array}$ & $\begin{array}{c}43.28 \\
(17.47)\end{array}$ & 886245.71 & 3375.26 & 85.71 \\
\hline \multirow{2}{*}{$\begin{array}{l}\text { Scenario } \\
\text { type }\end{array}$} & $\mathrm{A}$ & $\begin{array}{c}1013.85 \\
(1249.81)\end{array}$ & $\begin{array}{c}42.67 \\
(17.27)\end{array}$ & 853546.42 & 3331.06 & 85.21 \\
\hline & B & $\begin{array}{c}695.03 \\
(853.15)\end{array}$ & $\begin{array}{l}43.50 \\
(17.44)\end{array}$ & 937740.55 & 3433.44 & 86.42 \\
\hline
\end{tabular}

results establish a set of reference points to help assess the effectiveness of other formulations. The standard deviations of the objective function value and number of jobs done are shown parenthetically. Although, in general, we are unable to solve this formulation to optimality as indicated by the CPU times averaging close to the time limit, the lower objective function values than those in Table II in respective problems suggest that allowing deviation can markedly improve the effectiveness. This conjecture is also supported by 
TABLE IV

Summary of Results with the Two-Stage Heuristic with Fixed Scan-Off Angles

\begin{tabular}{|c|c|c|c|c|c|c|}
\hline Control Factor & Level & $\begin{array}{c}\text { Objective } \\
\text { Value }\end{array}$ & $\begin{array}{l}\text { Number of } \\
\text { Jobs Done }\end{array}$ & $\begin{array}{l}\text { Number of } \\
\text { Nodes }\end{array}$ & $\begin{array}{l}\text { CPU Time } \\
(\mathrm{sec})\end{array}$ & $\begin{array}{l}\text { Gap } \\
(\%)\end{array}$ \\
\hline \multirow{3}{*}{$\begin{array}{c}\text { Number of } \\
\text { jobs }\end{array}$} & 25.00 & $\begin{array}{c}456.82 \\
(267.39)\end{array}$ & $\begin{array}{l}21.37 \\
(1.38)\end{array}$ & 1170.59 & 0.95 & 0.00 \\
\hline & 50.00 & $\begin{array}{c}761.43 \\
(1040.89)\end{array}$ & $\begin{array}{l}45.17 \\
(1.94)\end{array}$ & 1502695.48 & 2700.90 & 35.94 \\
\hline & 75.00 & $\begin{array}{c}1088.62 \\
(1151.08)\end{array}$ & $\begin{array}{l}67.34 \\
(3.60)\end{array}$ & 1091768.92 & 3589.03 & 78.36 \\
\hline \multirow{4}{*}{$\begin{array}{l}\text { Number of } \\
\text { latency angles }\end{array}$} & 2.00 & $\begin{array}{c}1376.83 \\
(1359.35)\end{array}$ & $\begin{array}{c}43.90 \\
(19.09)\end{array}$ & 647131.98 & 2387.73 & 38.15 \\
\hline & 4.00 & $\begin{array}{c}910.73 \\
(996.92)\end{array}$ & $\begin{array}{c}44.48 \\
(19.11)\end{array}$ & 885459.35 & 2400.62 & 47.18 \\
\hline & 8.00 & $\begin{array}{c}495.59 \\
(192.23)\end{array}$ & $\begin{array}{c}44.42 \\
(18.70)\end{array}$ & 1179335.18 & 2244.02 & 43.21 \\
\hline & 16.00 & $\begin{array}{c}292.69 \\
(119.85)\end{array}$ & $\begin{array}{c}45.72 \\
(19.07)\end{array}$ & 748920.13 & 1355.46 & 23.86 \\
\hline \multirow{2}{*}{$\begin{array}{c}\text { Range of } \\
\text { processing times }\end{array}$} & & $\begin{array}{c}765.65 \\
(933.70)\end{array}$ & $\begin{array}{c}44.66 \\
(19.00)\end{array}$ & 888383.21 & 2153.64 & 40.46 \\
\hline & High & $\begin{array}{c}772.27 \\
(954.82)\end{array}$ & $\begin{array}{c}44.60 \\
(18.95)\end{array}$ & 842040.11 & 2040.27 & 35.74 \\
\hline \multirow{2}{*}{$\begin{array}{c}\text { Scenario } \\
\text { type }\end{array}$} & A & $\begin{array}{c}913.50 \\
(1061.24)\end{array}$ & $\begin{array}{c}44.15 \\
(18.78)\end{array}$ & 862610.29 & 2090.40 & 37.25 \\
\hline & B & $\begin{array}{c}624.42 \\
(784.42)\end{array}$ & $\begin{array}{l}45.10 \\
(19.16)\end{array}$ & 867813.03 & 2103.52 & 38.95 \\
\hline
\end{tabular}

the increased number of jobs done in Table III relative to that in Table II for corresponding problems.

Two-stage approximation strategies presented in Section IV are aimed to exploit the more modest computational requirements in the special case with no deviation. These strategies take the solution obtained for this special case as a starting point and seek to improve the overall objective of (16) as opposed to (12) by now allowing deviation. Table IV presents results with the two-stage approximation strategy presented in Section IVB1. This strategy solves the no deviation problem in the first stage and attempts to improve it in the second stage by allowing deviation. Unlike the original formulation this approach imposes the restriction that all of the previously scheduled jobs are to be processed with the scan-off angles assigned in the no-deviation solution. Once again the format of the table is similar to that of Tables II and III. With this strategy 211 of the 480 attempted instances are solved to optimality within the alloted time of $1 \mathrm{hr}$ in the improvement phase. Increasing the number of jobs increases the computational requirements in the improvement phase. Optimal solutions could be obtained for all 160 25-job instances in the second stage. However, the solution procedure stopped with a guaranteed optimality in the improvement phase for only 49 of the 16050 -job problems and 2 of the 16075 -job problems. After the improvement phase the average objective values improved by $24 \%, 32 \%$, and $38 \%$ for the 25-, 50-, and 75-job problems, respectively. Unlike in the special case with no deviation, increasing the number of scan-off angles resulted in a decrease in the computational requirements of the solution procedure in the improvement phase. This effect may be explained by the greater flexibility in assigning feasible starting times to the available jobs even in the first phase. For those problems with more alternative scan-off angles, a larger number of jobs were already processed in the initial solution and hence there was less work to be done in the improvement phase. In fact the percentage improvements in the objective value for the 2-, 4-, 8- and 16-angle problems are $42 \%, 34 \%, 13 \%$, and $2 \%$, respectively. Range of processing times does not appear to have marked effects in the improvement phase. Due to the larger number of high priority tasks in the type A scenario, its average objective function value is larger than that for the type B scenario. The scenario type does not have a significant effect on the computational requirements of the solution procedure. The standard deviations of the objective function value and number of jobs done are also shown parenthetically.

The results with the two-stage procedure of Section IVB1 are encouraging in terms of both computational efficiency and solution quality. Since preliminary experimentation indicated that the more flexible improvement strategy of Section IVB2 did not perform as efficiently, we do not report results with its use. The increased number of decisions due to the option of changing the scan-off angles of the 


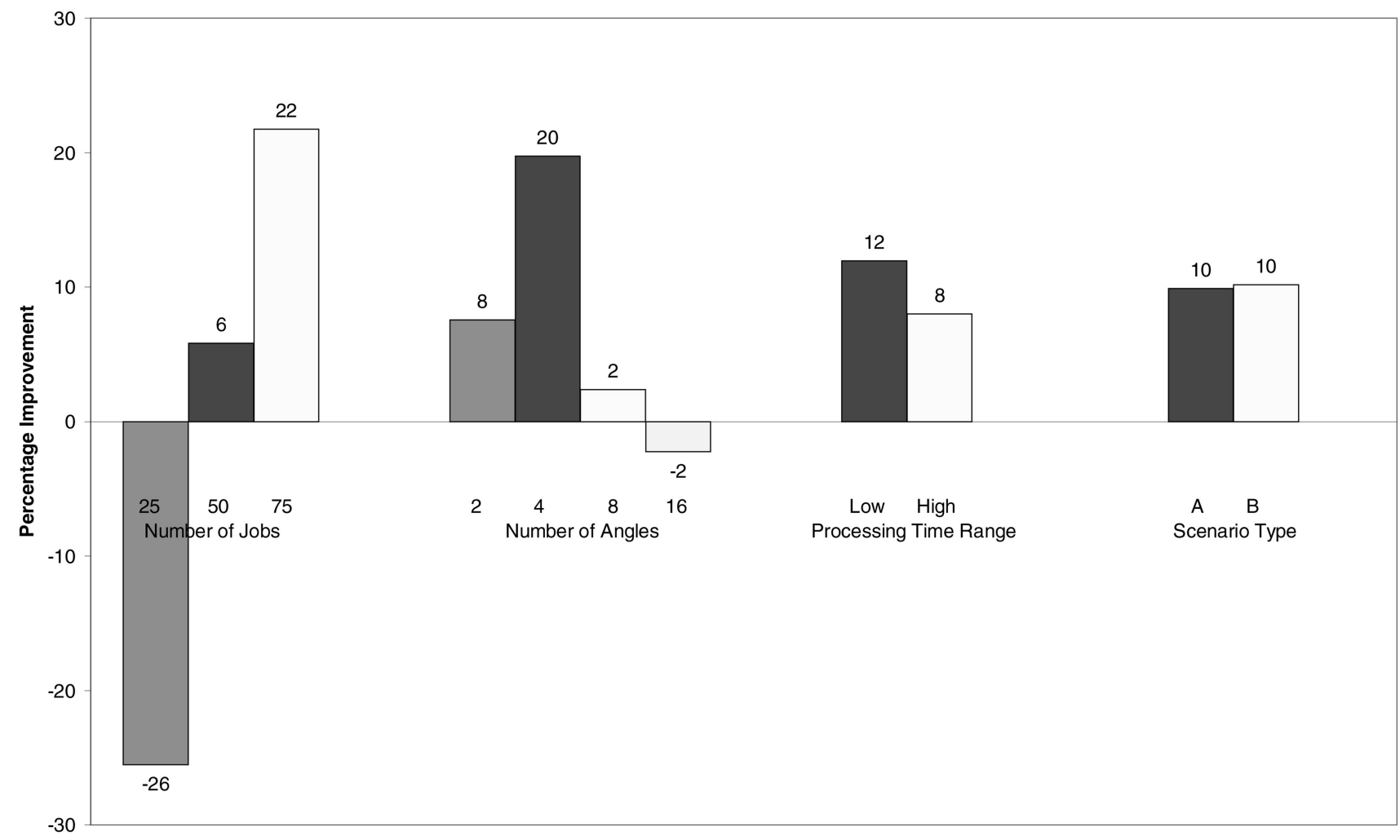

Fig. 5. Average percentage improvement obtained by using approximation procedure of Section IVB1 instead of the original formulation of Section IIIB.

jobs fixed in the first phase greatly increased the computational requirements.

As a next step in computational assessment, the performance of the two-step approximation procedure of Section IVB1 is analyzed relative to the best integer solutions obtained with the original strategy of Section IIIB. In particular we compare the results reported in Tables III and IV in Fig. 5. As the figure depicts, it becomes increasingly more advantageous to use the two-step approximation strategy as the problem size increases in terms of the number of jobs. Differences between the two-step approximation strategy and the original formulation do not appear to be significant for the 8- and 16-angle problems. As pointed out before, this may be due to the possibility of obtaining high quality initial integer feasible solutions with the no-deviation restriction when there are many different scan-off angles to choose from. There are only slight differences between the average performance of the two approaches in different processing time ranges and scenario types.

Finally, the effects of the proposed approach are assessed in the context of several scenarios considering both maneuvering and nonmaneuvering targets. We created scenarios with typical parameters. In these scenarios, the radar turns at a constant rate to complete a full rotation in $1.5 \mathrm{~s}$, and it uses a typical beam size. The false alarm rate is determined by rounding the numbers sampled from a Rayleigh distribution with $\sigma=0.798$ to the nearest integer, where the probability density function of the Rayleigh distribution is as follows.

$$
f(x, \sigma)=\frac{x}{\sigma^{2}} e^{-x^{2} / 2 \sigma^{2}} .
$$

In the nonmaneuvering scenario, a single target moves toward the radar from a $30-\mathrm{km}$ range at a speed of $200 \mathrm{~m} / \mathrm{s}$ until it approaches to a $15-\mathrm{km}$ distance. On the average one verification task arises alongside the tracking tasks of the target in each scan. In the maneuvering scenario, a single target with a constant altitude, approaching from a $20-\mathrm{km}$ range at a constant speed of $200 \mathrm{~m} / \mathrm{s}$, starts maneuvering with an acceleration of $5 \mathrm{~g}$ at a $15-\mathrm{km}$ distance for 20 scans, and then continues its movement at the same constant speed. Consequently, the number of tracking tasks in the maneuvering scenarios turns out to be between twice and three times as many as that in the nonmaneuvering case.

The number of scan-off angles is systematically varied as 4 and 8 in both maneuvering and nonmaneuvering scenarios. Fifty random instances are generated for each combination, resulting in a total of 200 instances with an average number of 42 jobs in each instance. These instances are solved first by the formulation that allows no deviation, and then by both the original formulation and the two-stage heuristic of Section IVB1. As in the random experiments, a CPU time limit of $1 \mathrm{hr}$ is imposed for all solution procedures, and the best integer solution 
TABLE V

Results for the Typical Scenarios

\begin{tabular}{|c|c|c|c|c|c|c|}
\hline Scenario Type & $\begin{array}{c}\text { Solution } \\
\text { Methodology }\end{array}$ & $\begin{array}{c}\text { Objective } \\
\text { Value }\end{array}$ & $\begin{array}{l}\text { Number of } \\
\text { Jobs Done }\end{array}$ & $\begin{array}{l}\text { Number of } \\
\text { Nodes }\end{array}$ & $\begin{array}{l}\text { CPU Time } \\
\quad(\mathrm{sec})\end{array}$ & $\begin{array}{l}\text { Gap } \\
(\%)\end{array}$ \\
\hline & No Deviation & $\begin{array}{c}4000.00 \\
(2469.95)\end{array}$ & $\begin{array}{l}29.78 \\
(1.45)\end{array}$ & 0.00 & 0.09 & 0.00 \\
\hline Nonmaneuvering & Original & $\begin{array}{c}3206.65 \\
(2357.22)\end{array}$ & $\begin{array}{l}34.30 \\
(2.13)\end{array}$ & 673972.66 & 2569.30 & 13.21 \\
\hline 4 angle & Two-stage & $\begin{array}{c}3310.75 \\
(2371.21)\end{array}$ & $\begin{array}{l}34.12 \\
(1.78)\end{array}$ & 16967.76 & 21.35 & 0.00 \\
\hline \multirow{3}{*}{ Nonmaneuvering } & No Deviation & $\begin{array}{c}3711.11 \\
(2502.86)\end{array}$ & $\begin{array}{l}31.92 \\
(1.37)\end{array}$ & 20.80 & 0.20 & 0.00 \\
\hline & Original & $\begin{array}{c}3120.62 \\
(2378.99)\end{array}$ & $\begin{array}{l}35.38 \\
(2.08)\end{array}$ & 1038858.68 & 3593.64 & 10.66 \\
\hline & Two-stage & $\begin{array}{c}3212.45 \\
(2384.98)\end{array}$ & $\begin{array}{l}34.78 \\
(1.75)\end{array}$ & 17977.62 & 14.84 & 0.00 \\
\hline \multirow{3}{*}{ Maneuvering } & No Deviation & $\begin{array}{c}4757.78 \\
(2363.30)\end{array}$ & $\begin{array}{l}29.44 \\
(1.50)\end{array}$ & 0.00 & 0.09 & 0.00 \\
\hline & Original & $\begin{array}{l}4144.56 \\
(2414.85)\end{array}$ & $\begin{array}{l}33.72 \\
(1.76)\end{array}$ & 532578.12 & 2118.70 & 11.50 \\
\hline & Two-stage & $\begin{array}{l}4222.94 \\
(2416.81)\end{array}$ & $\begin{array}{l}33.50 \\
(1.67)\end{array}$ & 281695.96 & 450.17 & 1.58 \\
\hline \multirow{3}{*}{ Maneuvering } & No Deviation & $\begin{array}{c}4048.78 \\
(2375.57)\end{array}$ & $\begin{array}{l}31.70 \\
(1.42)\end{array}$ & 96.96 & 0.27 & 0.00 \\
\hline & Original & $\begin{array}{c}3379.86 \\
(2278.37)\end{array}$ & $\begin{array}{l}35.08 \\
(2.17)\end{array}$ & 918719.36 & 3599.88 & 21.80 \\
\hline & Two-stage & $\begin{array}{c}3450.16 \\
(2284.47)\end{array}$ & $\begin{array}{l}34.94 \\
(2.18)\end{array}$ & 447970.38 & 655.70 & 2.43 \\
\hline
\end{tabular}

TABLE VI

Relative RMSE Values for the Typical Scenarios

\begin{tabular}{ccc}
\hline \hline Scenario Type & $\begin{array}{c}\text { Original } \\
\text { Formulation }\end{array}$ & $\begin{array}{c}\text { Two-Stage } \\
\text { Heuristic }\end{array}$ \\
\hline Nonmaneuvering 4 angle & 0.4269 & 0.4332 \\
Nonmaneuvering 8 angle & 0.3987 & 0.4013 \\
Maneuvering 4 angle & 1.0000 & 1.0290 \\
Maneuvering 8 angle & 0.6787 & 0.6709 \\
\hline
\end{tabular}

is reported when an algorithm fails to terminate within this alloted time. Table $\mathrm{V}$ shows the results for these instances in terms of the objective function and computational requirements of the scheduling problem. An optimum no-deviation solution could be found for all 200 instances mostly in fractions of a second. All 100 nonmaneuvering instances, 45 of the 50 maneuvering 4 -angle instances, and 44 of the 50 maneuvering 8 -angle instances were solved within $1 \mathrm{hr}$ by the two-stage heuristic as well. The original formulation could report the optimum solution only for 16 of the 4-angle nonmaneuvering, one of the 8-angle nonmaneuvering, 24 of the 4-angle maneuvering, and none of the 8-angle maneuvering instances. For the problems for which we could not secure an optimal solution within the alloted time, we use the best integer feasible solution found before termination. The average computational times also support the efficiency of the heuristic algorithm relative to the original formulation. As for the effectiveness, both the original formulation and the two-stage heuristic result in a considerable improvement over the zero-deviation solutions on the average. However, the average performance of the heuristic mechanism is only slightly worse than that of the original formulation within the imposed time limit. In order to observe the effects of the proposed scheduling mechanisms on the actual physical performance of the radar, the scheduling solutions provided by the original formulation and the two-stage heuristic are also tested within the radar simulation running 5000 random replications. Performance is measured in terms of the RMSE of azimuth and elevation angles. Although we are unable to provide the actual RMSE values obtained in this analysis, we provide the relative figures in Table VI. In particular the table shows the ratio of the average RMSE value in each scenario type to that obtained by the original formulation for the maneuvering 4-angle problems, which is the worst average RMSE value obtained from the original formulation. As was observed in Table $\mathrm{V}$ also, the worst average performances are seen with the maneuvering 4-angle problems followed by maneuvering 8-angle, nonmaneuvering 4-angle, and nonmaneuvering 8-angle problems in that order. Although the original formulation generally provides slightly better results than the two-stage heuristic, the differences do not seem to be large enough to justify the extra computational effort. Since observations made based on the objective function 
of the scheduling problem and those based on the average RMSE values are very similar, this analysis also supports that the scheduling solutions have a strong effect on the actual physical performance of the radar.

\section{CONCLUSION}

This paper studied a scheduling problem that appears as a subproblem in the design of a resource management system for a military surveillance radar. The radar functions as a single machine that receives a list of jobs available for processing by the end of a planning horizon. Each job has an associated priority, a deterministic processing time, an ideal starting time, and a set of available scan-off angles. The scan-off angles allow for introducing an offset from the actual starting time of a job. The problem involves the selection of the jobs to be processed and assignment of a starting time and scan-off angle pair to each selected job. The objective is to minimize a priority-based total penalty assessed due to not processing jobs exactly on their ideal starting times or not processing them at all. The paper proposes a mathematical formulation and two alternative heuristic approaches that rely on the exact solution of a special case in which no jobs are allowed to be processed with deviation. Computational results indicate that the general problem requires excessive CPU time even for the medium sized problems. On the other hand, the special case with no deviation can be solved efficiently by the proposed model. The two-stage heuristic approach tends to terminate in significantly shorter times than the original formulation and hence it may be adapted when time is of concern. Additionally, it provides an effective means in the investigation of the critical problem parameters as well as forming a reference point for future studies on this problem.

\section{ACKNOWLEDGMENT}

This study was performed during the development phase of a military radar KALKAN at ASELSAN. KALKAN was developed as a 3-D middle range search and track radar for fast and accurate detection and identification of low altitude air targets.

ASELSAN is the leading multi-product electronics company of Turkey that designs, develops, and manufactures modern electronic systems for military and professional clients.

The many valuable suggestions provided by Ugur Oguz throughout this research are very much appreciated.

\section{ELIF YAVUZTURK \\ REHIS Radar System \\ Engineering \\ ASELSAN Inc. \\ 06172 Yenimahalle \\ Ankara, Turkey}

\section{REFERENCES}

[1] Baker, K. R. and Scudder, G. D.

Sequencing with earliness and tardiness penalties: A review.

Operations Research, 38, 1 (1990), 22-36.

[2] Butler, J. M.

Multifunction radar tracking and control.

Ph.D. dissertation, University College London, UK, 1998.

[3] De Jong, J. L. and Van Norden, W. L.

Application of hybrid metaheuristics in sensor management.

Aerospace Science and Technology, 11, 4 (2007), 295-302.

[4] Ding, Z

A survey of radar resource management algorithms. In Canadian Conference on Electrical and Computer Engineering, vols. 1-4, 2008, 1489-1494.

[5] Elshafei, M., Sherali, H. D., and Smith, J. C. Radar pulse interleaving for multitarget tracking. Naval Research Logistics, 51, 1 (2004), 72-94.

[6] Feinberg, E. A., et al.

Sensor resource management for an airborne early warning radar.

In Proceedings of SPIE, Signal and Data Processing of Small Targets, vol. 4728, 2002, 145-156.

[7] Huizing, A. G. and Bloemen, A. A. F.

An efficient scheduling algorithm for a multifunction radar.

In IEEE International Symposium on Phased Array Systems and Technology_Revolutionary Developments in Phased Arrays, 1996, 359-364.

[8] Margialot, M. and Langholz, G.

Design and analysis of fuzzy schedulers using fuzzy Lyapunov synthesis.

Engineering Applications of Artificial Intelligence, 14, 2 (2001), 183-188.

[9] M'Hallah, R. and Bulfin, R. L.

Minimizing the weighted number of tardy jobs on a single machine with release dates.

European Journal of Operational Research, 176 (2007), 727-744.

[10] Miranda, S., et al.

Knowledge-based resource management for multifunction radar: A look at scheduling and task prioritization. IEEE Signal Processing Magazine, 23, 1 (2006), 66-76.

[11] Miranda, S., et al.

Comparison of scheduling algorithms for multifunction radar.

IET Radar Sonar and Navigation, 1, 6 (2007), 414-424.

[12] Miranda, S., et al.

Multifunction radar resource management

In F. Gini (Ed.), Knowledge-Based Radar Detection,

Tracking, and Classification, Hoboken, NJ: Wiley, 2006, 225-264.

[13] Moran, W., Suvorova, S., and Howard, S.

Sensor scheduling in radar.

In A. O. Hero, D. A. Castanon, D. Cochran, and K. Kastella (Eds.), Foundations and Applications of Sensor Management, New York: Springer, 2008, 221-256.

[14] Nemhauser, G. L. and Wolsey, L. A.

Integer and Combinatorial Optimization.

Hoboken, NJ: Wiley, 1998. 
Case study: Scheduling for a multi-function phased array radar system.

European Journal of Operational Research, 90 (1996), 13-25.

[16] Potts, C. N. and Van Wassenhove, L. N

Algorithms for scheduling a single machine to minimize the weighted number of late jobs.

Management Science, 34, 7 (1988), 843-858.

[17] Villarreal, P. J. and Bulfin, L.

Scheduling a single machine to minimize the weighted number of tardy jobs.

IIE Transactions, 15, 4 (1983), 337-343.

[18] Wintenby, J. and Krishnamurthy, V.

Hierarchical resource management in adaptive airborne surveillance radars.

IEEE Transactions on Aerospace and Electronic Systems, 42, 2 (2006), 401-419.

[19] Winter, E. and Baptiste, P. On scheduling a multifunction radar.

Aerospace Science and Technology, 11, 4 (2007), 289-294.

[20] Wolsey, L. A.

Integer Programming.

Hoboken, NJ: Wiley, 1998.

\section{Detecting Number of Coherent Signals in Array Processing by Ljung-Box Statistic}

We investigate the detection of the number of coherent signals in array processing. Because of the rank deficiency of the coherent signal covariance matrix, the conventional approaches, exploiting the structures of the eigenvalues of the signal covariance matrix, are not applicable to the coherent case. We propose to use the whiteness of the residue to detect the number of coherent signals. The steps of the proposed approach include estimation of the signal parameters in the model, subtraction of the estimated signals from the observed sequences, and computation of the whiteness measure by the Ljung-Box statistic. The rationale of the proposed scheme and related issues, including direction of arrival estimation, complexities, and performance, are discussed. The proposed approach is simulated and compared with well-known approaches, including the Akaike information criterion and the minimum description length (MDL) and its recent versions MDLB and MDLC, through numerical examples. The effectiveness of the proposed approach is verified by the numerical examples of 2-, 3-, and 4-source signals.

\section{INTRODUCTION}

There are various applications of detection and estimation utilizing sensor arrays. Finding the direction of arrival (DOA) of the signal impinging the sensor array is one of the fundamental functions to enable applications in radar, sonar, geophysics, and tracking systems [1].

In this work, we consider the far-field scenario, where the distance of the source signal is far greater than the array aperture and the source signals form planar wavefronts impinging the sensor arrays. In the multiple signal classification (MUSIC) algorithm, the covariance matrix of the received signals is decomposed into signal and noise subspaces. The properties of orthogonality among the signal and noise subspaces are exploited to estimate the DOAs. In [2-4], the maximum likelihood (ML) estimator is studied. In the ML approach, the DOAs are formulated as unknown parameters in the likelihood

Manuscript received May 31, 2010; revised March 3, 2011 and September 10, 2011; released for publication October 25, 2011.

IEEE Log No. T-AES/48/2/943849.

Refereeing of this contribution was handled by W. Blanding.

This work was supported by National Science Council of Taiwan, under grant number NSC 100-2221-E-001-004.

0018-9251/12/\$26.00 (c) 2012 IEEE 